\title{
Fair uplink bandwidth allocation and latency guarantee for mobile WiMAX using fuzzy adaptive deficit round robin
}

\begin{abstract}
The explosive demands of rich media applications with their diverse quality of service (QoS) requirements have continuously fuelled the needs for ever more powerful networks. One example of such a network is called WiMAX which is driven by WiMAX Forum based on IEEE 802.16 Wireless MAN standard. One of the issues that still remain open in WiMAX is the scheduling algorithm that goes to meet the QoS requirements. However, QoS provisioning of real-time and non real-time applications are frequently unstable due to insufficient allocation of bandwidth, which leads to degradation in latency guarantee and deterioration of overall system utilization. In this paper, an efficient bandwidth allocation algorithm for the uplink traffic in mobile WiMAX is proposed. Using intelligent systems approach upon the traffic service class information served by the base station (BS), an adaptive deadline-based scheme is designed. The scheme is fully dynamic to guarantee a specific maximum latency for real-time applications, besides improving fairness and throughput, giving due considerations to non real-time applications. The algorithm uses fuzzy logic control which is embedded in the scheduler; its function is to control and dynamically update the bandwidth required by the various service classes according to their respective priorities, maximum latency and throughput. Simulation results show that the proposed algorithm manages to optimize the overall system utilization while at the same time guarantee the maximum latency requirements for real-time traffic.
\end{abstract}

Keyword: Bandwidth allocation; IEEE 802.16; Fuzzy; QoS; Uplink 